\title{
Histo-blood group antigens as mediators of infections
}

Julie E. Heggelund ${ }^{1,3}$, Annabelle Varrot ${ }^{2}$, Anne Imberty $^{2}$, Ute Krengel ${ }^{1^{*}}$

${ }^{1}$ Department of Chemistry, University of Oslo, P.O. Box 1033, NO-0315 Blindern, Norway

${ }^{2}$ Centre de Recherches sur les Macromolécules Végétales (CERMAV), CNRS and Université Grenoble Alpes, 38000 Grenoble, France

${ }^{3}$ Present address: School of Biomedical Sciences, University of Leeds, LS2 9JT Leeds, UK \& School of Pharmacy, University of Oslo, P.O. Box 1068 Blindern, NO-0316 Oslo, Norway

*Corresponding author: Krengel, Ute (ute.krengel@kjemi.uio.no) 


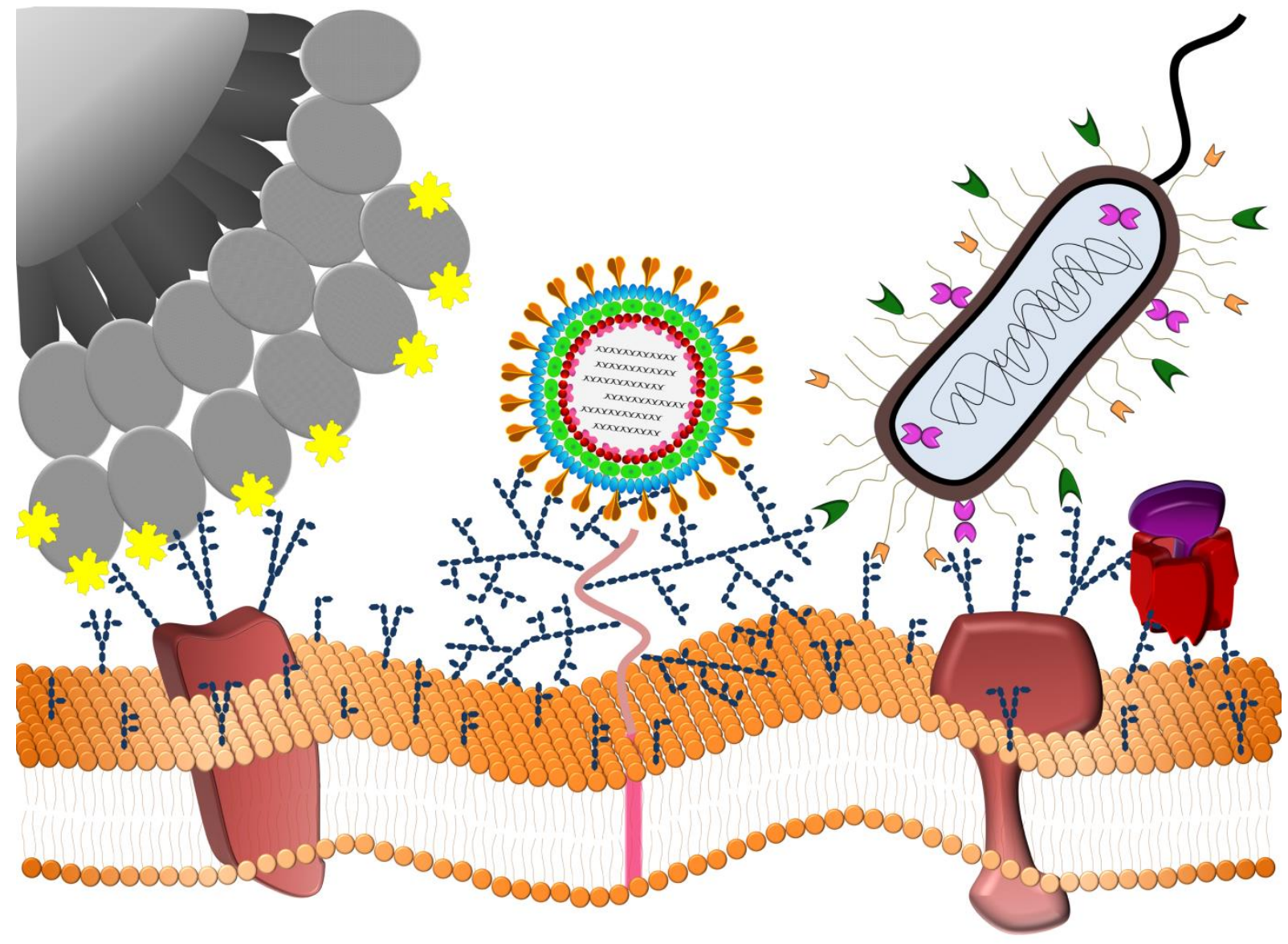

\section{Highlights}

- Cells are covered by a dense, protective glycan layer, the glycocalyx

- Viruses, bacteria and fungi exploit these glycans to gain entry to the host

- The mucus layer covering host cell epithelia sheds invaders

- Milk oligosaccharides can serve as protective decoys

- This review describes the structural mechanisms of this molecular arms race 


\begin{abstract}
The critical first step of a microbial infection is usually the attachment of pathogens to host cell glycans. Targets on host tissues are in particular the histo-blood group antigens, which are present in rich diversity in the mucus layer and on the underlying mucosa. Recent structural and functional studies have revealed significant new insight into the molecular mechanisms, explaining why individuals with certain blood groups are at increased risk of some infections. The most prominent example of blood-group-associated diseases is cholera, caused by infection with Vibrio cholerae. Many other microbial pathogens, e.g. Pseudomonas aeruginosa infecting the airways, and enterotoxigenic Escherichia coli (ETEC) causing traveler's diarrhea, also bind to histo-blood group antigens, but show a less clear correlation with blood group phenotype. Yet other pathogens, e.g. norovirus and Helicobacter pylori, recognize HBGAs differently depending on the strain. In all cases, milk oligosaccharides can aid the hosts' defenses, acting as natural receptor decoys, and antiinfectious therapy can be designed along similar strategies. In this review, we focus on important infections of humans, but the molecular mechanisms are of general relevance to a broad range of microbial infections of humans and animals.
\end{abstract}

\title{
Introduction
}

The battle between pathogens and their hosts is a constant race of evolution and adaptation. Once inside the host, either through the airways or the gastrointestinal tract, the pathogens must fight their way to the underlying cells. The mucus layer provides the first protective barrier of the underlying tissues (reviewed by [1]), and is essentially kept sterile by the constant secretion of mucus from goblet cells and the shedding of its top layer. This is true in particular for the colon, which has a second, inner mucus layer that is impermeable to bacteria. The mucus consists of a network of heavily glycosylated proteins, containing negatively charged carbohydrates and a significant amount of neutral, fucosylated sugars. The underlying mucous membrane, the mucosa, contains glycoconjugates including both lipids and proteins, resulting in a dense layer of glycans called the glycocalyx. This layer serves as a protective layer, helping to keep the pathogens at bay. However, many pathogens have evolved an ability to take advantage of these glycans, and use them as receptors for host cell adhesion or entry $[2,3]$. 
Especially well-characterized carbohydrate-based antigens are the histo-blood group antigens (HBGAs), which include the $A B H$ and Lewis antigens. The $A B H$ blood group system is named after the expression of antigens by red blood cells. HBGAs are also present on epithelial and endothelial cells throughout the body, and as soluble oligosaccharides in most body fluids except cerebrospinal fluid. Many pathogens use these antigens to adhere to host cells, and the interplay between pathogens and HBGAs is believed to promote the antigen diversity we see today $\left[4,5^{*}\right]$. Individuals have different active glycosyltransferases, leading to a large variety of oligosaccharides, whose expression varies greatly between tissues and even between tissue parts. A rich display of HBGAs is found in particular in the gastrointestinal tract and in the mucus layer covering it. This variation provides an advantage against pathogens, which rapidly evolve to maintain their competitive edge.

HBGAs are synthesized from precursor antigens and modified by the actions of different glycosyltransferases (Figure 1; reviewed by [4]). The simplest $\mathrm{ABH}$ antigens are the $\mathrm{H}$ antigens characteristic of blood group 0 , which can be converted into A or B antigens by the action of the $\mathrm{A}$ or $\mathrm{B}$ glycosyltransferases, respectively. $\mathrm{ABH}$ and precursor antigens can be fucosylated on GlcNAc, creating the Lewis antigens. The fucosyltransferase Secretor/FUT2 can add the so-called Secretor fucose. This enzyme is lacking in $20 \%$ of the European and North American population, resulting in the so-called non-secretor phenotype characterized by the absence of all $A B H$ antigens from mucus and secretions like saliva and human milk. Nevertheless, non-secretors can still express the simplest fucosylated Lewis antigens. In addition, symbiotic host microbes are known to contribute to intestinal fucosylation $\left[6^{*}\right]$.

The severity and susceptibility of many infectious diseases correlate with blood group phenotype [7]. So far, the understanding of the molecular mechanisms underlying this phenomenon has been very limited. This has changed recently with the determination of several high-resolution crystal structures of relevant protein-HBGA complexes. The gained insights are the topic of this review.

\section{Infections of the gastrointestinal tract}

Infection by enteric pathogens generally occurs through the fecal-oral route, caused by poor hygiene, consumption of contaminated food or water, or by exposure to infectious aerosols 
that are produced by vomiting. Some pathogens, such as Helicobacter pylori, are in addition transmitted through the oral-oral route, and others, e.g. Vibrio cholerae, can also survive in aquatic reservoirs, without any contact with feces.

\section{Viral infections}

Noroviruses (NoVs) and rotaviruses (RVs) are the two most important causes of acute gastroenteritis in humans. Both of them are 'non-enveloped' viruses, which means that they are encased by a protein capsid lacking a lipid envelope. Viruses replicate and evolve very rapidly, giving rise to a large number of different strains with different receptor profiles that enable them to conquer different niches (reviewed by $\left[8^{*}, 9^{*}, 10,11^{*}\right]$ ). Among their known cellular receptors are sialylated structures and HBGAs. Secretors are particularly susceptible to NoV and RV infections [12]. These individuals express HBGAs in their body fluids, mucus and gastrointestinal epithelial cells, therefore it is plausible that they are at higher risk of infection. While many children in the world are routinely vaccinated against RV infections, there is currently no vaccine against NoV infection.

The first examples of human pathogens, for which the molecular basis of blood group dependence was elucidated, are NoVs [13,14*]. They are single-stranded RNA viruses belonging to the Caliciviridae. Both major NoV genogroups, GI and GII, are known to infect humans and bind HBGAs in the high micromolar range [15]. GI includes the well-known Norwalk virus, causing winter-vomiting disease. This genogroup mainly targets individuals with blood group 0 , while people with blood group B have a lower risk of infection [16]. In contrast, GII NoVs do not discriminate between different blood groups $[17,18]$. The first Xray structure of a NoV was published in 1999 (PDB ID 1IHM [19]*). Its viral capsid contains dimeric $\mathrm{P}$ domains, which project out from the icosahedral shells and are responsible for the binding to the host cell receptors (Figure 2A,B). Intriguingly, the P domains (and in particular the receptor-binding P2 subdomains) have a much lower sequence identity than the overall genomes of GI and GII NoVs (approximately $25 \%$ (P2) compared to $50 \%$ overall $\left[11^{*}, 13\right]$ ). Moreover, the receptor binding sites are positioned at different locations at the $P$ dimerization interface and have distinct structural characteristics $\left[14^{*}, 8^{*}, 10\right]$ (Figure 2B-D). GI viruses bind HBGAs end-on and recognize mainly the terminal $\beta$-Gal residue of blood group $\mathrm{H}$-antigens (Figure $2 \mathrm{C}$ ). Additional interactions are found to the Secretor fucose, which include hydrophobic interactions to a conserved tryptophan residue, kept in place by cation- 
$\pi$ stacking to a histidine. In A or B antigens, the $\beta$-Gal binding site is instead occupied by the terminal $\alpha$-GalNAc or Gal residues characteristic for blood groups A and B, respectively, whereas the fucose residue is reoriented away from the conserved tryptophan into a secondary binding pocket (Figure $2 \mathrm{C}$ ). In $\mathrm{A}$ antigens, the $\mathrm{N}$-acetyl group of the terminal $\alpha$ GalNAc residue mimics the interactions of the Secretor fucose (Figure 2C), whereas $\alpha$-Gal, characteristic of B antigens, lacks these additional interactions and binds poorly to GI NoVs, explaining why individuals with blood group B experience protection. GII NoVs mainly recognize $\alpha$-fucose, present either as Secretor or Lewis fucose in a large variety of HBGAs [20-25] (Figure 2D). It is therefore not surprising that GII NoVs exhibit a broad ABH blood group profile.

Significantly less is known about RVs, both in terms of epidemiology and molecular mechanisms. RVs are double-stranded RNA viruses of the Reoviridae family, which form much larger viral particles than NoVs. VP8*, a subunit of the outer capsid protein VP4, is the functional equivalent of NoV's P domain (Figure 2E,F). VP8* has a galectin fold, a fold known to recognize $\beta$-Gal. However, in RVs the galactose binding site is blocked and a different site is involved in receptor recognition. For several decades, sialic acid has been known as the key cellular receptor of RVs, whereas the binding of HBGAs to certain RV strains was discovered only recently $[26,27]$. Nevertheless, the limited data that exist suggest that secretors are significantly more susceptible to RV infections than non-secretors [12], indicating that HBGAs may indeed be functional RV receptors. In 2012, a crystal structure of VP8* was reported in complex with a blood group A-trisaccharide [28*], occupying the same site that binds sialic acid in other RV strains. The major interactions were to the $\alpha$-GalNAc characteristic of blood group $A$ and additional interactions to $\beta$-Gal, whereas the Secretorfucose faced away from the HBGA binding site (Figure 2F). Nevertheless, glycan array data showed that this residue strongly enhances receptor binding [28*], which may be due to intramolecular conformational stabilization. Overall, binding specificity varies greatly between different RV strains, and even strains binding the same antigens exhibit considerable variation in molecular recognition $[26,27,29]$. Differences in the width of the receptor cleft may play a role in the different binding properties [10]. The structural adaptability of the VP8* binding site, including receptor-induced conformational changes, 
may furthermore lie at the heart of receptor-release prior to cell entry [22*] and of interspecies transmission $[27,30]$.

\section{Bacterial infections}

Many bacteria use lectins to attach to host cells (reviewed in this issue by Moonens \& Remaut [31*]). One notable example is Helicobacter pylori, responsible for inducing stomach ulcers and gastric cancer. More than half of the world population is infected with this bacterium, which was declared a class I carcinogen by the WHO in 1994 . The most virulent $H$. pylori strains mediate adhesion to the human host via the Blood group Antigen Binding Adhesin, BabA [32], which is present in large amounts in H. pylori membranes and facilitates the bacterial colonization of the stomach mucosa. Different $H$. pylori strains exhibit distinct blood group profiles [33]: in populations with high incidence of the blood group 0 phenotype, such as the natives of South America, specialist species predominate, while in Europe and the USA, which have a more diverse blood group pattern, generalist species of $H$. pylori are able to bind all three $\mathrm{ABH}$ blood group antigens. The different binding profiles are correlated with the presence of BabA variants with different specificities for ABH/Lewis glycans. Moonens et al. recently solved the crystal structures of several of these BabA variants in complexes with various HBGAs, revealing the molecular basis of this phenomenon [34**]. BabA's interaction with host receptors is three-pronged (Figure $3 \mathrm{~A}$ ): (i) Its main anchor is the Secretor fucose, which is bound by a loop stabilized by a redox-sensitive disulfide bond called Çys-clasped Loop CL2, involving hydrogen bonds to several highly conserved amino acid residues. ii) The Asp-Ser-Ser triad of the Diversification Loop DL2 specifically binds type 1 glycan receptors, ensuring tropism for the foveolar epithelium, which covers the inside of the stomach. iii) Blood group A/B-specific $\alpha$-GalNAc/Gal residues bind to a shallow pocket in the DL1 region. O-specific $H$. pylori strains exhibit a distinct sequence variation in this loop, where a proline in combination with a bulky residue (such as Asn, Asp, or Leu) sterically interferes with generalist binding. Additional phenotypes are the $A B$ specialists, which bind more tightly to $A / B$-specific glycans due to additional interactions of the terminal $\alpha-G a I N A c / G a l$ residues with DL1, and the inverse specialists, which bind A/Brather than O-specific glycans despite exhibiting the bulky Pro-Leu sequence, since a nonfunctional DL2 loop allows for a slight rotation that prevents the steric clash (Figure 3A). 
Cholera is arguably the most well-known example of blood-group-dependent diseases. For 50 years, individuals with blood group $\mathrm{O}$ have been known to experience more severe symptoms than those with other blood groups [35], although paradoxically they are less likely to be infected. The main culprit is the cholera toxin (CT), which after gaining entry to host cells, hijacks the host's own endogenous pathways to trigger the opening of ion channels, inducing the massive secretory diarrhea typical of the disease. Secretors experience protection from cholera [36], which is in contrast to many other diseases. In the past few years, great strides were made in understanding the blood group association of cholera on a detailed molecular level. The primary receptor of the CT is the GM1 ganglioside, which binds to the toxin with nanomolar affinity [37]. That CT can also bind to HBGAs or analogs of these has been shown only recently [38,39]. Studies of chimera of the receptorbinding subunits of CT and the homologous heat-labile toxin (LT) from enterotoxigenic Escherichia coli (ETEC) indicated that the toxins may harbor a second binding site $[40,41]$, for HBGAs, that is spatially distinct from the GM1 binding site (Figure 3B). Subsequently, the crystal structure of LT was determined in complex with a blood-group-A-specific human milk oligosaccharide (HMO) resembling A-Lewis ${ }^{y}$, sparking a discussion as to why ETEC infections nevertheless do not show a strong blood group association [42*]. This is probably due to interference with LPS: in contrast to the $\mathrm{CT}$, which is secreted as a soluble toxin, LT remains attached to outer membrane vesicles via LPS. Indications are that the binding sites for HBGAs and LPS overlap, interfering with LT binding to HBGAs [43], which would explain the lack of blood group association for ETEC infections. For cholera, conflicting data were published regarding the binding of two natural CT variants to HBGAs and HMO analogs $[38,39,44]$. The breakthrough came last year with the high-resolution structures of respective CT-complexes [45**] (Figure 3B) and supporting cell biological experiments [46], explaining all previous findings. Unexpectedly the blood group A determinant (and its HMO analog) bound in the opposite orientation compared to LT, showing that subtle amino acid differences in the secondary binding site can considerably modulate binding. Another unexpected result was the binding of $\mathrm{H}$-determinants (Lewis ${ }^{y}$ and its $\mathrm{HMO}$-analog) in two alternative orientations. This rationalized the stronger binding of O-specific glycans compared to A- and B-determinants, where the A/B-specific $\alpha$-GalNAc/Gal residues can only be accommodated in one of the orientations. Again a fucose residue (generally the Lewis fucose) was shown to play the central role in binding, connecting two adjacent CT subunits. 
Even though binding affinities to HBGAs are only in the millimolar range and hence significantly weaker than to GM1 $\left[37,45^{* *}\right]$, CT is capable of cooperatively binding these ligands with all five of its receptor-binding subunits, permitting high-avidity interaction with its target tissue. Stronger binding of the toxin to O-specific compared to A/B-specific glycans is consistent with the observed blood group profile of cholera. What remains to be further investigated is why secretors are protected.

Other enteric pathogens known to bind HBGAs are Campylobacter jejuni [47] and Salmonella enterica serotype Typhimurium [48], although structural data regarding these interactions are not yet available.

\section{Infections of the airways}

While healthy airway mucus helps to expel microorganisms by coughing and sneezing, pathogenic mucus, as encountered in cystic fibrosis or chronic obstructive pneumonia, becomes a niche for chronic infection, leading to morbidity and mortality. The glycosylation of mucus changes during the course of pathologies, although it is not yet clear whether the changes are due to the disease itself, to associated inflammation or due to the presence of microbes [49-51]. Modifications in fucosylation, sulfatation and sialylation are generally observed in cystic fibrosis, but with different trends depending on $\mathrm{N}$ - or $\mathrm{O}$-glycosylation. Opportunistic pathogens, often associated with hospital environments, include bacteria such as Pseudomonas aeruginosa and members of the Burkholderia complexes, and airborne fungi such as Aspergillus fumigatus. All of these pathogens bind to HBGAs, but so far only $P$. aeruginosa has been investigated for correlation between blood group and host susceptibility.

$P$. aeruginosa infection is the leading cause of death of cystic fibrosis patients, and also affects immunocompromised patients. Conflicting data exist regarding its possible correlation with blood group phenotype. While the analysis of gene polymorphism in cystic fibrosis patients did not reveal any correlation between severity of $P$. aeruginosa infection and $\mathrm{ABH}$, secretor or Lewis genotypes [52], other studies indicated a stronger susceptibility to $P$. aeruginosa sepsis in children with blood group B [53] and a correlation of external otitis with blood groups A or B [54]. P. aeruginosa interacts with host glycans mainly via two 
soluble lectins, the $\alpha$-galactose specific LecA and the fucose-specific LecB, which have both been structurally characterized [55]. The LecA target is likely the aGal1-4Gal disaccharide present on globotriaosylceramide (Gb3) and blood group $P^{k}$ and $P_{1}$ antigens [56]. LecA binding to Gb3 triggers the uptake of the bacterium into airway cells in vitro [57]. LecB has a very strong affinity for fucose due to the rare chelation of two calcium ions, and its bestknown ligand is the Lewis ${ }^{a}$ epitope. Two recent studies investigated the variations of LecB genes in more than 200 bacterial strains originating from natural or clinical environments $[58,59 * *$. Sequence analysis revealed two main clusters that could be identified as PAO1like and PA14-like. The fucose binding site is conserved in all strains, with $13 \%$ variations of sequences between PAO1 and P14. Crystal structures and affinity analysis of two variants from PA14 [59**] and PA7 [58] strains confirmed that binding to fucose is conserved, indicating the importance of LecB for these bacteria. Furthermore, multivalent epitope presentation was shown to be critical. For example, the LecB variant from the pathogenic PA14 strain binds with very high affinity $\left(K_{d}=25 \mathrm{nM}\right)$ to a biantennary $N$-glycan presenting two blood group $\mathrm{H}$ epitopes, corresponding to an affinity that is 10 -fold higher than for isolated $\mathrm{H}$-type 2 oligosaccharide $\left(K_{\mathrm{d}}=200 \mathrm{nM}\right)$ [59**].

The fucose-specific six-blade $\beta$-propeller fold represents another lectin family identified in lung pathogens, i.e. B. ambifaria (BambL) [60] and A. fumigatus (AFL or FleA) [61]. The biological role of these lectins is not yet entirely clear. For example, AFL was demonstrated to contribute to inflammation [61], but also stimulated macrophage killing, thus protecting the host $\left[62^{*}\right]$. The 6 -blade $\beta$-propeller fold is formed by a tandem repeat of one single chain in fungi or by trimerization of a much shorter chain in bacteria (Figure $4 A, B$ ). In AFL, the six binding sites exhibit minor differences in amino acid composition with variations in oligosaccharide preference as observed by soaking of crystals in a mixture of oligosaccharides [63*]. Nevertheless, both bacterial and fungal family members bind fucose in a unique conserved binding mode involving hydrogen bonds between an arginine and the $\mathrm{O} 4$ hydroxyl or ring oxygens, and between a Glu/GIn residue and the $\mathrm{O} 3$ and $\mathrm{O} 4$ hydroxyls as well as a $\mathrm{CH}-\pi$ interaction with a Tyr/Trp residue (Figure 4C-F). The general preference for blood group $\mathrm{O}$ (H-type 2) and Lewis ${ }^{y}$ was rationalized through molecular modeling [64], showing that the binding pockets are well oriented for multivalent binding of terminal aFuc1-2Gal epitopes (Figure 4D). Apart for its preference for Lewis ${ }^{\mathrm{y}}$, AFL binds to all 
fucosylated oligosaccharides, and therefore to all tissues expressing Lewis and $A B H$ antigens. The $\alpha$ Fuc1-3GIcNAc disaccharide present in Lewis ${ }^{x / y}$ is also recognized, and analysis of the crystal complexes led to the surprising observation that the "closed" conformation, observed in solution, solid state and modeling [65], is forced into an "open" conformation when entering the binding sites (Figure 4E-F). A conserved aromatic residue appears to be responsible for guiding the fucose into the binding site by separating the fucose and galactose rings that are stacked together in solution $\left[66^{* *}\right]$. Opening of the canonical Lewis ${ }^{x}$ conformation apparently involves the transient distortion of the central $\mathrm{N}$-acetylglucosamine ring, as shown by crystal structures of another family member, Ralstonia solanacearum lectin (RSL), in complexes with Lewis $^{\mathrm{x}}$ and sialyl-Lewis ${ }^{\mathrm{x}}$, combined with extensive molecular dynamics simulations [66**].

\section{Fighting back: glycans, glycocompounds and glycomimetics as anti-infectious}

\section{agents}

Anti-infectious strategies can be based on competition with the attachment of pathogens to HBGAs on target tissues. Such protection is provided naturally by human milk, which contains a large variety of fucosylated and sialylated oligosaccharides (HMOs). These soluble oligosaccharides protect newborns from infections in a dual fashion, as anti-microbials and prebiotics $\left[6^{*}, 67^{*}\right]$. Interesting examples, where structural information is available, are cholera $\left[44,45^{* *}\right]$ and norovirus infections [68]. Building on the accumulated structural knowledge of lectin-oligosaccharide interactions, it has been possible to design glycomimetics with increased affinities for lectin receptors, achieving a stronger competition effect [69]. Classical strategies are centered on optimizing the binding energy between protein and carbohydrates, i.e. increasing the enthalpy of binding by maximizing the number of hydrogen bonds and/or hydrophobic contacts, and decreasing the entropy contribution by reducing the flexibility of the oligosaccharide. For example, the careful design of the aglycone (=non-sugar) part of a fucose derivative significantly increased the selectivity for its target B. cenocepacia BambL [70]. Similarly, a fucopyranoside glycomimetic inhibitor of $A$. fumigatus FleA was shown to inhibit binding and phagocytosis of conidia by macrophages $\left[62^{*}\right]$. 
Another strategy is to design multivalent ligands that simultaneously bind to several binding sites of a multivalent receptor, resulting in a strong gain in avidity (=apparent multivalent affinity) $\left[71^{*}, 72^{*}\right]$. A successful example is a divalent galactoside ligand of $P$. aeruginosa LecA, which was able to lower the cellular invasiveness of the bacteria by up to $90 \%$ by chelating two neighboring receptor binding sites [73]. Other examples are the prevention of human immunodeficiency virus (HIV) transmission by glycodendrimers [74] and the design of multi-valent inhibitors against bacterial toxins $[72 *]$. Based on high-throughput screening of compounds libraries, both in vitro and in silico, it is also possible to identify noncarbohydrate glycomimetics able to compete in carbohydrate binding sites, as accomplished for noroviruses [75]. Yet another possibility is the design of HBGA-blocking antibodies as therapeutic agents ([76] and references therein).

\section{Conclusions and Perspective}

A large variety of human pathogens recognize blood group antigens. These include viruses, bacteria and fungi, which employ viral coat proteins, microbial adhesins, soluble lectins and toxins to invade and conquer their hosts [2]. In this review, we focus on human infections of the gastrointestinal tract and the airways. A common microbial strategy is to use HBGA binding for attachment and entry to the host cells. Secretors, who display these antigens on tissues exposed to the external environment and on their mucus, nurture symbionts [6*] but are also disproportionally affected by infectious diseases (however, as described below, hosts have their own measures to force selective pressures onto microbes, e.g. through blood group polymorphism, to prevent the eradication of entire populations $\left.\left[4,5^{*}\right]\right)$. Another microbial strategy is to employ HBGA-binding toxins. Not discussed in this review is the molecular mechanism of parasitical malaria infection, where adhesins facilitate erythrocyte rosetting by binding primarily to blood group A antigens [77], explaining why individuals with blood group A are most severely affected by this disease. This has consequences on a population level, due to natural selection and evolution. The Ganges delta, for example, where both malaria and cholera are endemic, has a unique blood group profile, with the highest percentage of blood group B in the world. Blood group polymorphism is an important asset for surviving in this world [4]. Microbes respond by evolving many strains with different binding properties, but the hosts can fight back. Of particular importance is the continuously renewing mucus layer, which nurtures symbionts and helps expel 
pathogens $\left[1,6^{*}\right]$. Another trick is human milk, which mimics HBGAs, serving as nondegradable receptor decoy protecting human infants $\left[67^{*}\right]$. The high fucose-content of human milk is likely of particular importance for the protective effect. Modified oligosaccharides, glycomimetics and dendrimers work in a similar way, with potential as medications and prophylactics [69,72*]. Enveloped viruses like influenza virus and HIV take their host's membrane coats along on their journey to a new victim. This provides them with disguise, but can also elicit immune reactions against foreign blood groups. The molecular arms race is on, and we are now beginning to understand its molecular underpinnings, providing us with new tools to fight disease.

\section{Acknowledgements}

The authors gratefully acknowledge funding from the University of Oslo, from CNRS, from the University Grenoble Alpes through Glyco@Alps (ANR-15-IDEX-02) and from ANR through Labex Arcane (ANR-11-LABX-0003-01). 


\section{References}

1. Johansson MEV, Ambort D, Pelaseyed T, Schütte A, Gustafsson JK, Ermund A, Subramani DB, Holmén-Larsson JM, Thomsson KA, Bergström JH, et al.: Composition and functional role of the mucus layers in the intestine. Cell Mol Life Sci 2011, 68:36353641.

2. Imberty A, Varrot A: Microbial recognition of human cell surface glycoconjugates. Curr Opin Struct Biol 2008, 18:567-576.

3. Sperandio B, Fischer N, Sansonetti PJ: Mucosal physical and chemical innate barriers: Lessons from microbial evasion strategies. Semin Immunol 2015, 27:111-118.

4. Marionneau S, Cailleau-Thomas A, Rocher J, Le Moullac-Vaidye B, Ruvoën N, Clément $M$, Le Pendu J: ABH and Lewis histo-blood group antigens, a model for the meaning of oligosaccharide diversity in the face of a changing world. Biochimie 2001, 83:565573.

5. Bishop JR, Gagneux P: Evolution of carbohydrate antigens--microbial forces shaping host glycomes? Glycobiology 2007, 17:23R-34R.

*Thought-provoking review analyzing the molecular arms race underlying the evolution of carbohydrate antigens to the host glycomes existing today.

6. Pickard JM, Chervonsky AV: Intestinal fucose as a mediator of host-microbe symbiosis. J Immunol 2015, 194:5588-5593.

* Review describing the important role of fucose as mediator of host-microbe symbiosis and the relevance of gut fucosylation to human disease.

7. Cooling L: Blood groups in infection and host susceptibility. Clin Microbiol Rev 2015, 28:801-870.

8. Tan $M$, Jiang $X$ : Histo-blood group antigens: a common niche for norovirus and rotavirus. Expert Rev Mol Med 2014, 16:e5.

* Review giving a good and well-structured overview over HBGA recognition by NoVs and RVs, and implications for epidemiology and evolution. A comparison to other HBGA-binding pathogens, e.g. Helicobacter pylori, is also included.

9. Ströh $\sqcup$, Stehle T: Glycan engagement by viruses: receptor switches and specificity. Annu Rev Virol 2014, 1:285-306. 
*Interesting review on glycan receptor switching by viruses focusing on cases where highresolution structural data are available, such as NoV and RV infections.

10. Venkataram Prasad BV, Shanker S, Hu L, Choi J-M, Crawford SE, Ramani S, Czako R, Atmar RL, Estes MK: Structural basis of glycan interaction in gastroenteric viral pathogens. Curr Opin Virol 2014, 7:119-127.

11. de Graaf M, van Beek J, Koopmans MPG: Human norovirus transmission and evolution in a changing world. Nat Rev Microbiol 2016, 14:421-433.

* Review giving a good and well-structured overview over human norovirus transmission, pathogenesis and evolution.

12. Kambhampati A, Payne DC, Costantini V, Lopman BA: Host genetic susceptibility to enteric viruses: a systematic review and metaanalysis. Clin Infect Dis 2016, 62:11-18.

13. Cao S, Lou Z, Tan M, Chen Y, Liu Y, Zhang Z, Zhang XC, Jiang X, Li X, Rao Z: Structural basis for the recognition of blood group trisaccharides by norovirus. J Viro/ 2007, 81:5949-5957.

14. Choi J-M, Hutson AM, Estes MK, Venkataram Prasad BV: Atomic resolution structural characterization of recognition of histo-blood group antigens by Norwalk virus. Proc Nat Acad Sci U S A 2008, 105:9175-9180.

*First structural analysis of the blood group $O$ preference of Norwalk virus (GI) and comparison to genogroup II NoVs, explaining their different blood group profiles.

15. Hansman GS, Shahzad-UI-Hussan S, McLellan JS, Chuang G-Y, Georgiev I, Shimoike T, Katayama K, Bewley CA, Kwong PD: Structural basis for norovirus inhibition and fucose mimicry by citrate. J Virol 2012, 86:284-292.

16. Hutson AM, Atmar RL, Graham DY, Estes MK: Norwalk virus infection and disease is associated with ABO histo-blood group type. J Infect Dis 2002, 185:1335-1337.

17. Huang P, Farkas T, Marionneau S, Zhong W, Ruvoën-Clouet N, Morrow AL, Altaye M, Pickering LK, Newburg DS, LePendu J, et al.: Noroviruses bind to human ABO, Lewis, and secretor histo-blood group antigens: identification of 4 distinct strain-specific patterns. J Infect Dis 2003, 188:19-31.

18. Rockx BHG, Vennema H, Hoebe CJPA, Duizer E, Koopmans MPG: Association of histoblood group antigens and susceptibility to norovirus infections. J Infect Dis 2005, 191:749-754. 
19. Venkataram Prasad BV, Hardy ME, Dokland T, Bella J, Rossmann MG, Estes MK: X-ray crystallographic structure of the Norwalk virus capsid. Science 1999, 286:287-290.

*First structure of a NoV revealing atomic-level details.

20. Chen Y, Tan M, Xia M, Hao N, Zhang XC, Huang P, Jiang X, Li X, Rao Z: Crystallography of a Lewis-binding norovirus, elucidation of strain-specificity to the polymorphic human histo-blood group antigens. PLoS Pathog 2011, 7:e1002152.

21. Hansman GS, Biertümpfel C, Georgiev I, McLellan JS, Chen L, Zhou T, Katayama K, Kwong PD: Crystal structures of GII.10 and GII.12 norovirus protruding domains in complex with histo-blood group antigens reveal details for a potential site of vulnerability. $J$ Virol 2011, 85:6687-6701.

22. Shanker S, Choi J-M, Sankaran B, Atmar RL, Estes MK, Venkataram Prasad BV: Structural analysis of histo-blood group antigen binding specificity in a norovirus GII.4 epidemic variant: implications for epochal evolution. J Virol 2011, 85:8635-8645.

*Structural characterization of HBGA-complexes of several GII NoV strains show that HBGA binding sites can be conformationally flexible, which is of potential importance for receptor release before entry into host cells as well as for evolutionary dynamics.

23. Koromyslova AD, Leuthold MM, Bowler MW, Hansman GS: The sweet quartet: Binding of fucose to the norovirus capsid. Virology 2015, 483:203-208.

24. Singh BK, Leuthold MM, Hansman GS: Human noroviruses' fondness for histo-blood group antigens. J Virol 2015, 89:2024-2040.

25. Schroten H, Hanisch F-G, Hansman GS: Human norovirus interactions with histo-blood group antigens and human milk oligosaccharides. J Virol 2016, 90:5855-5859.

*Good short overview over the current knowledge of human norovirus interactions with HBGAs and HMOs, supported by a nice figure.

26. Huang P, Xia M, Tan M, Zhong W, Wei C, Wang L, Morrow A, Jiang X: Spike protein VP8* of human rotavirus recognizes histo-blood group antigens in a type-specific manner. J Virol 2012, 86:4833-4843.

27. Ramani S, Hu L, Venkataram Prasad BV, Estes MK: Diversity in rotavirus-host glycan interactions: a "sweet" spectrum. Cell Mol Gastroenterol Hepatol 2016, 2:263-273.

28. Hu L, Crawford SE, Czako R, Cortes-Penfield NW, Smith DF, Le Pendu J, Estes MK, Venkataram Prasad BV: Cell attachment protein VP8* of a human rotavirus 
specifically interacts with A-type histo-blood group antigen. Nature 2012, 485:256259.

*First article demonstrating and structurally characterizing A-type HBGA binding to RVs.

29. Böhm R, Fleming FE, Maggioni A, Dang VT, Holloway G, Coulson BS, von Itzstein $M$, Haselhorst T: Revisiting the role of histo-blood group antigens in rotavirus host-cell invasion. Nat Commun 2015, 6:5907.

30. Yu X, Mishra R, Holloway G, von Itzstein M, Coulson BS, Blanchard H: Substantial receptor-induced structural rearrangement of rotavirus VP8*: Potential implications for cross-species infection. Chembiochem 2015, 16:2176-2181.

31. Moonens $\mathrm{K}$, Remaut $\mathrm{H}$ : Evolution and structural dynamics of bacterial glycan binding adhesins. Curr Opin Struct Biol 2017, 44:48-58.

*Good overview over the structural mechanisms employed by glycan-binding bacterial adhesins, including dynamic adaptations in space and time. Focus on UPEC and $H$. pylori adhesins.

32. Ilver D, Arnqvist A, Ögren J, Frick I-M, Kersulyte D, Incecik ET, Berg DE, Covacci A, Engstrand L, Borén T: Helicobacter pylori adhesin binding fucosylated histo-blood group antigens revealed by retagging. Science 1998, 279:373-377.

33. Aspholm-Hurtig M, Dailide G, Lahmann M, Kalia A, Ilver D, Roche N, Vikström S, Sjöström R, Lindén S, Bäckström A, et al.: Functional adaptation of BabA, the $\boldsymbol{H}$. pylori ABO blood group antigen binding adhesin. Science 2004, 305:519-522.

34. Moonens K, Gideonsson P, Subedi S, Bugaytsova J, Romað E, Mendez M, Nordén J, Fallah M, Rakhimova L, Shevtsova A, et al.: Structural Insights into polymorphic ABO glycan binding by Helicobacter pylori. Cell Host Microbe 2016, 19:55-66.

**Molecular basis of the polymorphic blood group profile of Helicobacter pylori infections, elucidated by a comprehensive analysis of its BabA adhesin in crystal complexes with various HBGA determinants. Implications for redox-active pharmaceutical treatment are also discussed.

35. Barua D, Paguio AS: ABO blood groups and cholera. Ann Hum Biol 1977, 4:489-492.

36. Chaudhuri A, DasAdhikary CR: Possible role of blood-group secretory substances in the aetiology of cholera. Trans R Soc Trop Med Hyg 1978, 72:664-665. 
37. Turnbull WB, Precious BL, Homans SW: Dissecting the cholera toxin-ganglioside GM1 interaction by isothermal titration calorimetry. J Am Chem Soc 2004, 126:10471054.

38. Heggelund JE, Haugen E, Lygren B, Mackenzie A, Holmner $\AA$, Vasile F, Reina JJ, Bernardi A, Krengel U: Both El Tor and classical cholera toxin bind blood group determinants. Biochem Biophys Res Commun 2012, 418:731-735.

39. Mandal PK, Branson TR, Hayes ED, Ross JF, Gavín JA, Daranas AH, Turnbull WB: Towards a structural basis for the relationship between blood group and the severity of EI Tor cholera. Angew Chem Int Ed Engl 2012, 51:5143-5146.

40. Ångström J, Bäckström M, Berntsson A, Karlsson N, Holmgren J, Karlsson K-A, Lebens M, Teneberg S: Novel carbohydrate binding site recognizing blood group A and B determinants in a hybrid of cholera toxin and Escherichia coli heat-labile enterotoxin B-subunits. J Biol Chem 2000, 275:3231-3238.

41. Holmner $\AA$, Lebens $M$, Teneberg S, Ångström J, Ökvist M, Krengel U: Novel binding site identified in a hybrid between cholera toxin and heat-labile enterotoxin: $1.9 \AA$ crystal structure reveals the details. Structure 2004, 12:1655-1667.

42. Holmner $\AA$, Askarieh G, Ökvist M, Krengel U: Blood group antigen recognition by Escherichia coli heat-labile enterotoxin. J Mol Biol 2007, 371:754-764.

*First structural analysis of the heat-labile toxin from ETEC probing interaction with HBGAs (here: complex with a blood group A analog of human milk), as a basis for a thorough discussion of the molecular mechanisms of cholera and ETEC infections.

43. Mudrak B, Rodriguez DL, Kuehn MJ: Residues of heat-labile enterotoxin involved in bacterial cell surface binding. J Bacteriol 2009, 191:2917-2925.

44. Vasile F, Reina JJ, Potenza D, Heggelund JE, Mackenzie A, Krengel U, Bernardi A: Comprehensive analysis of blood group antigen binding to classical and El Tor cholera toxin B-pentamers by NMR. Glycobiology 2014, 24:766-778.

45. Heggelund JE, Burschowsky D, Bjørnestad VA, Hodnik V, Anderluh G, Krengel U: Highresolution crystal structures elucidate the molecular basis of cholera blood group dependence. PLoS Pathog 2016, 12:e1005567.

**Molecular mechanisms of cholera blood group dependence, elucidated by a comprehensive structural analysis of cholera toxin-HBGA and -HMO complexes, including 
high-resolution crystal structures (to $1 \AA$ ) of classical and El Tor biotypes and quantitative binding data from SPR analysis.

46. Kuhlmann FM, Santhanam S, Kumar P, Luo Q, Ciorba MA, Fleckenstein JM: Blood group O-dependent cellular responses to cholera toxin: parallel clinical and epidemiological links to severe cholera. Am J Trop Med Hyg 2016, 95:440-443.

47. Ruiz-Palacios GM, Cervantes LE, Ramos P, Chavez-Munguia B, Newburg DS:

Campylobacter jejuni binds intestinal $\mathrm{H}(0)$ antigen (Fuca1, 2Galß1, 4GICNAC), and fucosyloligosaccharides of human milk inhibit its binding and infection. J Biol Chem 2003, 278:14112-14120.

48. Chessa D, Winter MG, Jakomin M, Bäumler AJ: Salmonella enterica serotype Typhimurium Std fimbriae bind terminal $\alpha(1,2)$ fucose residues in the cecal mucosa. Mol Microbiol 2009, 71:864-875.

49. Xia B, Royall JA, Damera G, Sachdev GP, Cummings RD: Altered O-glycosylation and sulfation of airway mucins associated with cystic fibrosis. Glycobiology 2005, 15:747-775.

50. Venkatakrishnan V, Thaysen-Andersen M, Chen SCA, Nevalainen H, Packer NH: Cystic fibrosis and bacterial colonization define the sputum $\mathrm{N}$-glycosylation phenotype. Glycobiology 2015, 25:88-100.

51. Jeffries JL, Jia J, Choi W, Choe S, Miao J, Xu Y, Powell R, Lin J, Kuang Z, Gaskins HR, et al.: Pseudomonas aeruginosa pyocyanin modulates mucin glycosylation with sialylLewis $^{x}$ to increase binding to airway epithelial cells. Mucosal Immunol 2016, 9:10391050.

52. Taylor-Cousar JL, Zariwala MA, Burch LH, Pace RG, Drumm ML, Calloway H, Fan H, Weston BW, Wright FA, Knowles MR: Histo-blood group gene polymorphisms as potential genetic modifiers of infection and cystic fibrosis lung disease severity. PLoS One 2009, 4:e4270.

53. Kuo K-C, Kuo H-C, Huang L-T, Lin C-S, Yang S-N: The clinical implications of ABO blood groups in Pseudomonas aeruginosa sepsis in children. J Microbiol Immunol Infect 2013, 46:109-114.

54. Steuer MK, Hofstädter F, Pröbster L, Beuth J, Strutz J: Are ABH antigenic determinants on human outer ear canal epithelium responsible for Pseudomonas aeruginosa infections? ORL J Otorhinolaryngol Relat Spec 1995, 57:148-152. 
55. Imberty A, Wimmerová M, Mitchell EP, Gilboa-Garber N: Structures of the lectins from Pseudomonas aeruginosa: insights into the molecular basis for host glycan recognition. Microbes Infect 2004, 6:221-228.

56. Gilboa-Garber N, Sudakevitz D, Sheffi M, Sela R, Levene C: PA-I and PA-II lectin interactions with the $\mathrm{ABO}(\mathrm{H})$ and $\mathrm{P}$ blood group glycosphingolipid antigens may contribute to the broad spectrum adherence of Pseudomonas aeruginosa to human tissues in secondary infections. Glycoconj J 1994, 11:414-417.

57. Eierhoff T, Bastian B, Thuenauer R, Madl J, Audfray A, Aigal S, Juillot S, Rydell GE, Müller S, de Bentzmann S, et al.: A lipid zipper triggers bacterial invasion. Proc Natl Acad Sci U S A 2014, 111:12895-12900.

58. Boukerb AM, Decor A, Ribun S, Tabaroni R, Rousset A, Commin L, Buff S, DoléansJordheim A, Vidal S, Varrot A, et al.: Genomic rearrangements and functional diversification of $L e c A$ and $L e c B$ lectin-coding regions impacting the efficacy of glycomimetics directed against Pseudomonas aeruginosa. Front Microbiol 2016, 7:811.

59. Sommer R, Wagner S, Varrot A, Nycholat CM, Khaledi A, Häussler S, Paulson JC, Imberty $A$, Titz $A$ : The virulence factor LecB varies in clinical isolates: consequences for ligand binding and drug discovery. Chem Sci 2016, 7:4990-5001.

**Evidence that analysis of lectin sequence and specificity should not be limited to the laboratory microorganisms, but should explore the diversity of bacterial strains, especially in hospital environments.

60. Audfray A, Claudinon J, Abounit S, Ruvöen-Clouet N, Larson G, Smith DF, Wimmerová M, Le Pendu J, Römer W, Varrot A, et al.: Fucose-binding lectin from opportunistic pathogen Burkholderia ambifaria binds to both plant and human oligosaccharidic epitopes. J Biol Chem 2012, 287:4335-4347.

61. Houser J, Komarek J, Kostlanova N, Cioci G, Varrot A, Kerr SC, Lahmann M, Balloy V, Fahy JV, Chignard M, et al.: A soluble fucose-specific lectin from Aspergillus fumigatus conidia-structure, specificity and possible role in fungal pathogenicity. PLoS One 2013, 8:e83077.

62. Kerr SC, Fischer GJ, Sinha M, McCabe O, Palmer JM, Choera T, Lim FY, Wimmerova M, Carrington SD, Yuan S, et al.: FleA expression in Aspergillus fumigatus is recognized 
by fucosylated structures on mucins and macrophages to prevent lung infection. PLoS Pathog 2016, 12:e1005555.

* Study of host defense mechanisms triggered by $A$. fumigatus lectin and evidence that a fucopyranoside glycomimetic can inhibit the immune clearance mechanisms triggered by the lectin.

63. Houser J, Komarek J, Cioci G, Varrot A, Imberty A, Wimmerova M: Structural insights into Aspergillus fumigatus lectin specificity: AFL binding sites are functionally nonequivalent. Acta Crystallogr D Biol Crystallogr 2015, 71:442-453.

*Deciphering the puzzle of the specificity of the six binding sites of $A$. fumigatus lectin by SPR, and clever use of oligosaccharides for crystallization.

64. Topin J, Arnaud J, Sarkar A, Audfray A, Gillon E, Perez S, Jamet H, Varrot A, Imberty A, Thomas A: Deciphering the glycan preference of bacterial lectins by glycan array and molecular docking with validation by microcalorimetry and crystallography. PLoS One 2013, 8:e71149.

65. Zierke M, Smieško M, Rabbani S, Aeschbacher T, Cutting B, Allain FH-T, Schubert M, Ernst B: Stabilization of branched oligosaccharides: Lewis ${ }^{x}$ benefits from a nonconventional C-H”OO hydrogen bond. J Am Chem Soc 2013, 135:13464-13472.

66. Topin J, Lelimousin M, Arnaud J, Audfray A, Pérez S, Varrot A, Imberty A: The hidden conformation of Lewis $x$, a human histo-blood group antigen, is a determinant for recognition by pathogen lectins. ACS Chem Biol 2016, 11:2011-2020.

**First evidence that a bacterial lectin can induce a non-canonical conformation of a Lewis epitope upon binding.

67. Etzold S, Bode L: Glycan-dependent viral infection in infants and the role of human milk oligosaccharides. Curr Opin Virol 2014, 7:101-107.

* Review over the current knowledge about how HMOs protect breast-fed infants from viral diseases like RV, NoV and HIV infections.

68. Weichert S, Koromyslova A, Singh BK, Hansman S, Jennewein S, Schroten H, Hansman GS: Structural basis for norovirus inhibition by human milk oligosaccharides. J Virol 2016, 90:4843-4848.

69. Sattin S, Bernardi A: Glycoconjugates and glycomimetics as microbial anti-adhesives. Trends Biotechnol 2016, 34:483-495. 
70. Richichi B, Imberty A, Gillon E, Bosco R, Sutkeviciute I, Fieschi F, Nativi C: Synthesis of a selective inhibitor of a fucose binding bacterial lectin from Burkholderia ambifaria. Org Biomol Chem 2013, 11:4086-4094.

71. Wittmann V, Pieters RJ: Bridging lectin binding sites by multivalent carbohydrates. Chem Soc Rev 2013, 42:4492-4503.

* Review on the achievement of biologically relevant binding affinities and selectivities by multivalency in protein-carbohydrate interactions.

72. Branson TR, Turnbull WB: Bacterial toxin inhibitors based on multivalent scaffolds. Chem Soc Rev 2013, 42:4613-4622.

* Review discussing the major classes of multivalent toxin inhibitors investigated to date, including glycopolymers, glycodendrimers, tailored glycoclusters and inhibitors exploiting templated assembly.

73. Novoa A, Eierhoff T, Topin J, Varrot A, Barluenga S, Imberty A, Römer W, Winssinger N: A LecA ligand identified from a galactoside-conjugate array inhibits host cell invasion by Pseudomonas aeruginosa. Angew Chem Int Ed Engl 2014, 53:8885-8889.

74. Garcia-Vallejo JJ, Koning N, Ambrosini M, Kalay H, Vuist I, Sarrami-Forooshani R, Geijtenbeek TBH, van Kooyk Y: Glycodendrimers prevent HIV transmission via DCSIGN on dendritic cells. Int Immunol 2013, 25:221-233.

75. Ali ES, Rajapaksha H, Carr JM, Petrovsky N: Norovirus drug candidates that inhibit viral capsid attachment to human histo-blood group antigens. Antiviral Res 2016, 133:1422.

76. Venkataram Prasad BV, Shanker S, Muhaxhiri Z, Deng L, Choi J-M, Estes MK, Song Y, Palzkill T, Atmar RL: Antiviral targets of human noroviruses. Curr Opin Virol 2016, 18:117-125.

77. Vigan-Womas I, Guillotte M, Juillerat A, Hessel A, Raynal B, England P, Cohen JH, Bertrand O, Peyrard T, Bentley GA, et al.: Structural basis for the ABO blood-group dependence of Plasmodium falciparum rosetting. PLoS Pathog 2012, 8:e1002781.

78. Settembre EC, Chen JZ, Dormitzer PR, Grigorieff N, Harrison SC: Atomic model of an infectious rotavirus particle. EMBO J 2011, 30:408-416.

79. Merritt EA, Kuhn P, Sarfaty S, Erbe JL, Holmes RK, Hol WGJ: The 1.25 Å resolution refinement of the cholera toxin B-pentamer: evidence of peptide backbone strain at the receptor-binding site. J Mol Biol 1998, 282:1043-1059. 
80. Sixma TK, Kalk KH, van Zanten BAM, Dauter Z, Kingma J, Witholt B, Hol WGJ: Refined structure of Escherichia coli heat-labile enterotoxin, a close relative of cholera toxin. J Mol Biol 1993, 230:890-918. 


\section{Figure Legends}

Figure 1: Biosynthetic pathways of type 1 and type 2 human histo-blood group antigens (HBGAs). Carbohydrate symbols are shown in the standard schematic representation introduced by the Consortium for Functional Glycomics. H-type 1 ( $\alpha 3$-linkage) and H-type 2 ( $\alpha 4)$ precursors are fucosylated by $\alpha-1,2$-fucosyltransferase (FUT2) or $\alpha-1,3 / 4$ fucosyltransferase (FUT3/4), adding the Secretor fucose and Lewis fucose, respectively. Blood group $\mathrm{H}$ determinants (characteristic of blood group 0 ) can subsequently be modified by the action of $A$ or $B$ glycosyltransferases, which add an $\alpha$-GalNAc or $\alpha$-Gal residue to $\beta$ Gal, to give rise to blood group A or B antigens, respectively. Non-secretors lack the FUT2 gene and can therefore only synthesize the smallest Lewis antigens, Lewis ${ }^{\mathrm{a}}$ and Lewis ${ }^{\mathrm{x}}$.

Figure 2: HBGA recognition by noro- and rotaviruses. (A) Structure of NoV capsid (PDB 1IHM [19*]). (B) Structure of individual protruding P domains (dimers). Gray - P domain of GI NoV in complexes with H-pentasaccharide (green sticks; PDB 2ZL6 [14*]) and A-trisaccharide (cyan sticks; PDB 2ZL7 [14*]). Green/slate - GII P domain bound to A-trisaccharide (yellow sticks; PDB 3SLD $\left.\left[22^{*}\right]\right)$. (C) Top view of the GI and GII P domain complexes shown in (B) and close-up view of GI NoV interaction with H-type 1 pentasaccharide (green sticks, with fucose in magenta) and the corresponding A-trisaccharide (cyan sticks, including fucose). Important amino acid and carbohydrate residues are labeled, $\mathrm{H}$-type 1 residues in italics. $\mathrm{H}$-bonds are indicated by red dashed lines, and the hydrophobic interaction to Trp with yellow filled circles. Note that the acetamido group of GalNAc characteristic of $A$ antigens partially mimics the interactions of the H-type 1 fucose. (D) Top view of the GII P domain complex shown in (B), and details of GII NoV interaction with A-trisaccharide (yellow sticks, fucose in magenta). The virus is anchored to the fucose residue, while the GaINAc residue provides only limited contacts. (E) RV capsid structure (PDB 4V7Q [78*]) with protruding VP4 spikes (teal). (F) Top domains of VP4, with A-trisaccharide (yellow sticks, with fucose in magenta) bound to the VP8* domain. The close-up view shows details of VP8* A-trisaccharide interaction (PDB 4DRV [28]). Carbohydrate residues are labeled, the fucose is highlighted in magenta and $\mathrm{H}$ bonds are indicated with red dashes. Main interactions are to the GalNAc residue characteristic of $A$ antigens. 
Figure 3: HBGA-interactions of selected bacterial pathogens of the gastrointestinal tract. (A) Molecular basis of $H$. pylori adaptation to human HBGA polymorphism. The $H$. pylori adhesin BabA binds to HBGAs with three loops (DL1, CL2, DL2), displaying significant variation between different strains. For example, ABO generalists can accommodate the Gal (green sticks) or GalNAc residues (not shown) characteristic of B or A antigens, respectively, whereas bulky residues in DL1 prevent HBGA binding due to steric interference, leading to O-specialists (close-up view). This panel is reproduced with permission from [31*]. (B) Molecular basis of cholera blood group dependence. The culprit is the cholera toxin (CT; cartoon representation), which binds to HBGAs (box) and GM1 (green sticks; PDB 3CHB [79]). Several CT structures are superimposed. The CT A subunit is colored purple (PDB 1LTS [80]), the CT B pentamer blue, gray and magenta (PDB 3CHB [78]). A close-up view shows the detailed interactions of $\mathrm{CT}$ with the $\mathrm{H}$-tetrasaccharide (white sticks; PDB 5ELB [45**]) and the A-pentasaccharide (colored sticks; PDB 3ELD [45**]). The color code is the same as for the standard schematic representations shown below. Note that H-tetra can bind in two orientations, whereas the A-pentasaccharide (BGA or HMO) binds in a single orientation (Se Fuc, Secretor fucose; Le Fuc, Lewis fucose).

Figure 4: HBGA-pathogen interactions of selected airway infections. (A,B) Overall representations of the 6-bladed $\beta$-propeller folds of BambL in complex with the H-type 2 determinant (A, PDB 3ZZV [60]) and AFL (FleA) with a blood group A determinant (B, PDB 4AH4 [63]). Coloring is by protein chain or blade, respectively. (C) Close-up view of the fucose binding site 3 in AFL. (D-F) Surface representations of the binding of H-type 2 (D; PDB 3ZZV [60]) and Lewis ${ }^{x}$ (E, PDB 3ZW1 [60]) determinants in the intramolecular binding site of BamBL, and Lewis ${ }^{y}$ (F, PDB 4D4U [63]) in binding site 3 of AFL. 


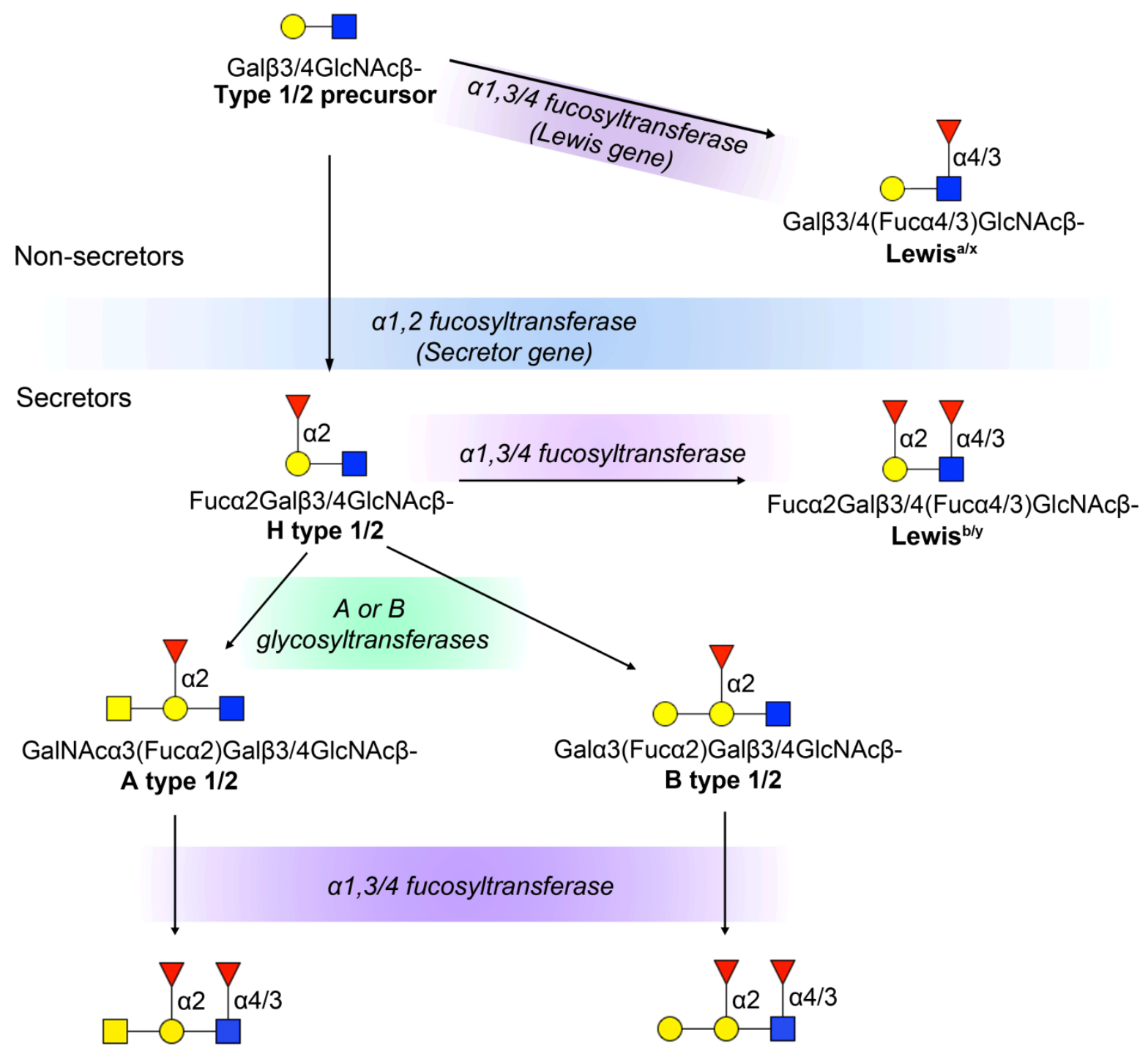

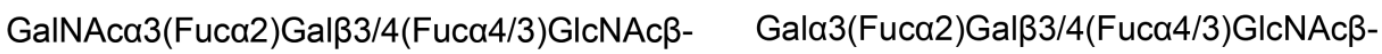

A Lewis ${ }^{\mathrm{b} / \mathrm{y}}$

B Lewis Lly $^{\mathrm{b}}$

Figure 1 Biosynthetic pathways of type 1 and type 2 human histo-blood group antigens (HBGAs). Carbohydrate symbols are shown in the standard schematic representation introduced by the Consortium for Functional Glycomics. H-type 1 ( $\alpha 3$-linkage) and H-type 2 ( $\alpha 4)$ precursors are fucosylated by $\alpha-1,2$-fucosyltransferase (FUT2) or $\alpha-1,3 / 4$ fucosyltransferase (FUT3/4), adding the Secretor fucose and Lewis fucose, respectively. Blood group $\mathrm{H}$ determinants (characteristic of blood group $\mathrm{O}$ ) can subsequently be modified by the action of A or B glycosyltransferases, which add a GalNAc or Gal residue to $\beta$-Gal, to give rise to blood group A or B antigens, respectively. Non-secretors lack the FUT2 gene and can therefore only synthesize the smallest Lewis antigens, Lewis ${ }^{\mathrm{a}}$ and Lewis ${ }^{\mathrm{x}}$. 
A

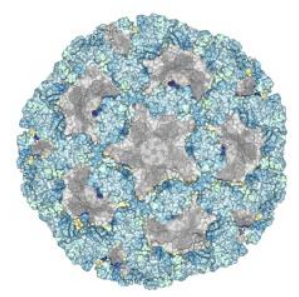

C

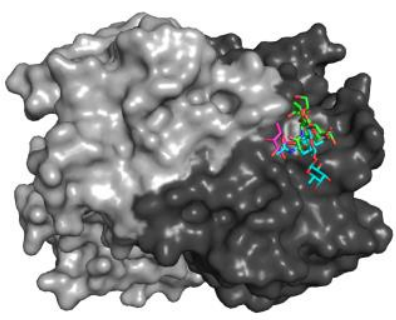

D

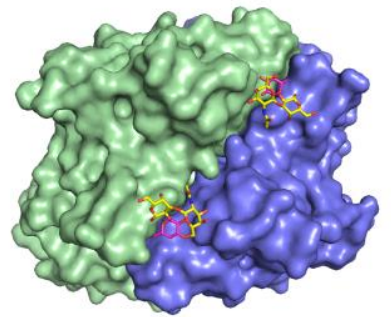

B
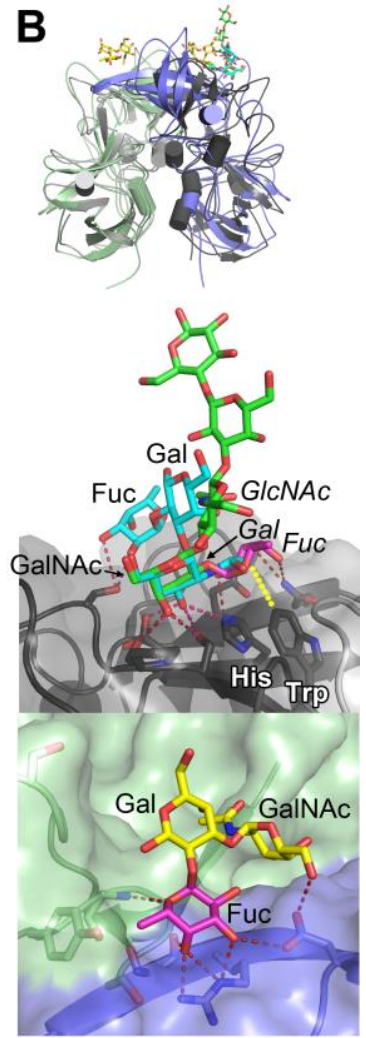

E
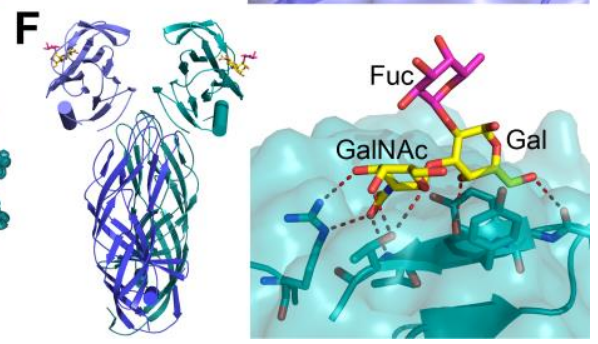

Figure 2 HBGA recognition by noro- and rotaviruses. (A) Structure of NoV capsid (PDB 1IHM [19*]). (B) Structure of individual protruding P domains (dimers). Gray - P domain of GI NoV in complexes with H-pentasaccharide (green sticks; PDB 2ZL6 [14*]) and A-trisaccharide (cyan sticks; PDB 2ZL7 [14*]). Green/slate - GII P domain bound to A-trisaccharide (yellow sticks; PDB 3SLD [22*]). (C) Top view of the GI and GII P domain complexes shown in (B) and close-up view of GI NoV interaction with $\mathrm{H}$-type 1 pentasaccharide (green sticks, with fucose in magenta) and the corresponding A-trisaccharide (cyan sticks, including fucose). Important amino acid and carbohydrate residues are labeled, $\mathrm{H}$-type 1 residues in italics. $\mathrm{H}$-bonds are indicated by red dashed lines, and the hydrophobic interaction to Trp with yellow filled circles. Note that the acetamido group of GalNAc characteristic of $A$ antigens partially mimics the interactions of the $\mathrm{H}$-type 1 fucose. (D) Top view of the GII P domain complex shown in (B), and details of GII NoV interaction with A-trisaccharide (yellow sticks, fucose in magenta). The virus is anchored to the fucose residue, while the GalNAc residue provides only limited contacts. (E) RV capsid structure (PDB 4V7Q [78*]) with protruding VP4 spikes (teal). (F) Top domains of VP4, with A-trisaccharide (yellow sticks, with fucose in magenta) bound to the VP8* domain. The close-up view shows details of VP8* A-trisaccharide interaction (PDB 4DRV [28]). Carbohydrate residues are labeled, the fucose is highlighted in magenta and $\mathrm{H}$ bonds are indicated with red dashes. Main interactions are to the GalNAc residue characteristic of $A$ antigens. 

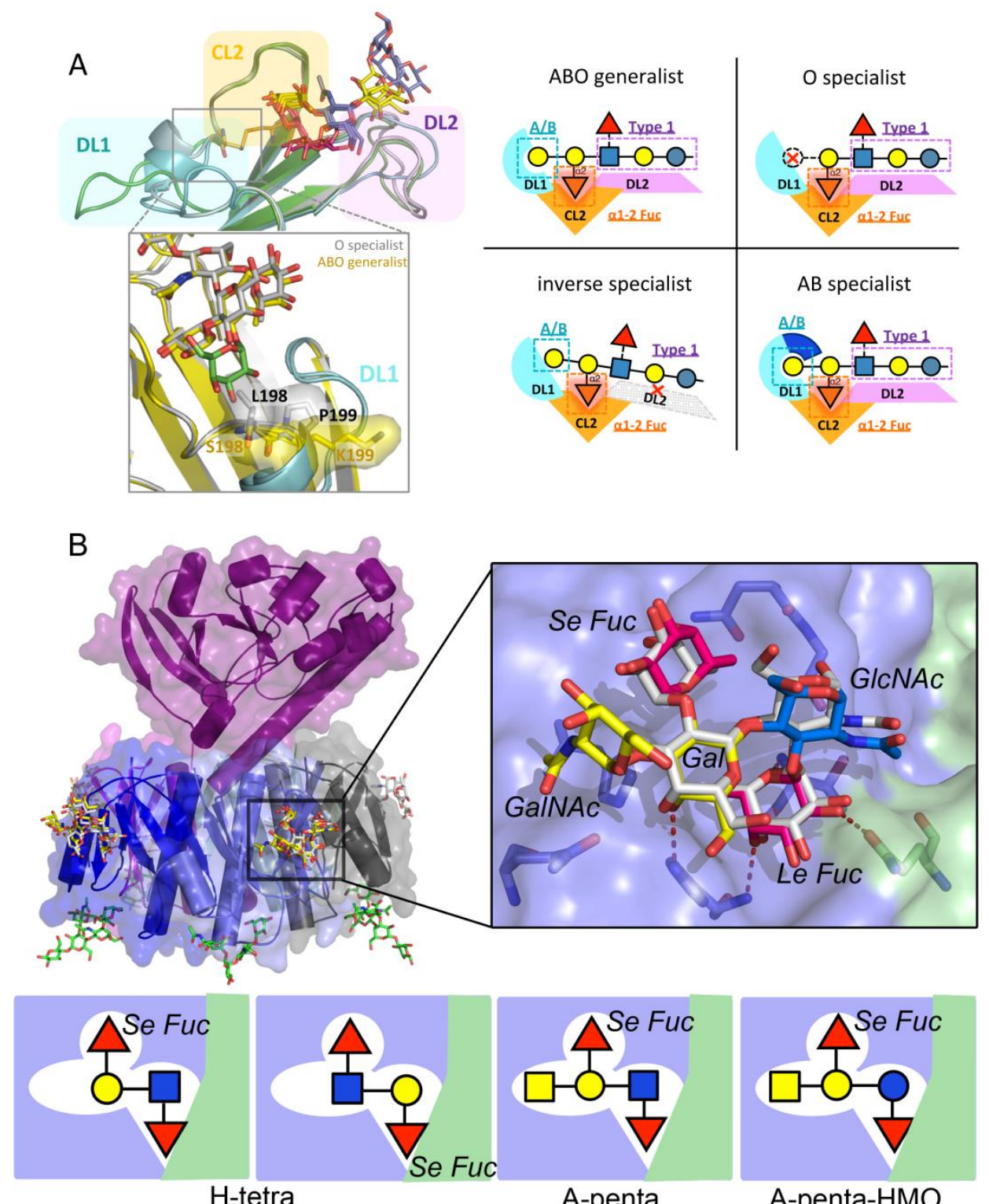

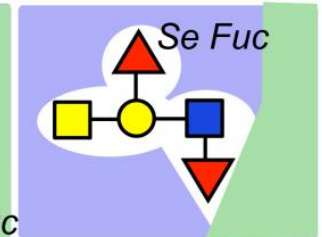

A-penta

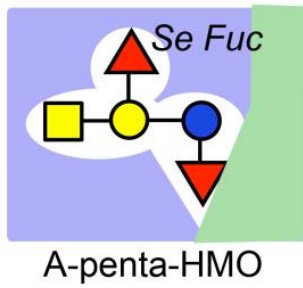

Figure 3 HBGA-interactions of selected bacterial pathogens of the gastrointestinal tract. (A) Molecular basis of $H$. pylori adaptation to human HBGA polymorphism. The $H$. pylori adhesin BabA binds to HBGAs with three loops (DL1, CL2, DL2), displaying significant variation between different strains. For example, $A B O$ generalists can accommodate the Gal (green sticks) or GalNAc residues (not shown) characteristic of $B$ or $A$ antigens, respectively, whereas bulky residues in DL1 prevent HBGA binding due to steric interference, leading to O-specialists (close-up view). This panel is reproduced with permission from [31*]. (B) Molecular basis of cholera blood group dependence. The culprit is the cholera toxin (CT; cartoon representation), which binds to HBGAs (box) and GM1 (green sticks; PDB 3CHB [79]). Several CT structures are superimposed. The CT A subunit is colored purple (PDB 1LTS [80]), the CT B pentamer blue, gray and magenta (PDB 3CHB [78]). A close-up view shows the detailed interactions of CT with the H-tetrasaccharide (white sticks; PDB 5ELB [45**]) and the A-pentasaccharide (colored sticks; PDB 3ELD [45**]). The color code is the same as for the standard schematic representations shown below. Note that H-tetra can bind in two orientations, whereas the A-pentasaccharide (BGA or HMO) binds in a single orientation (Se Fuc, Secretor fucose; Le Fuc, Lewis fucose). 

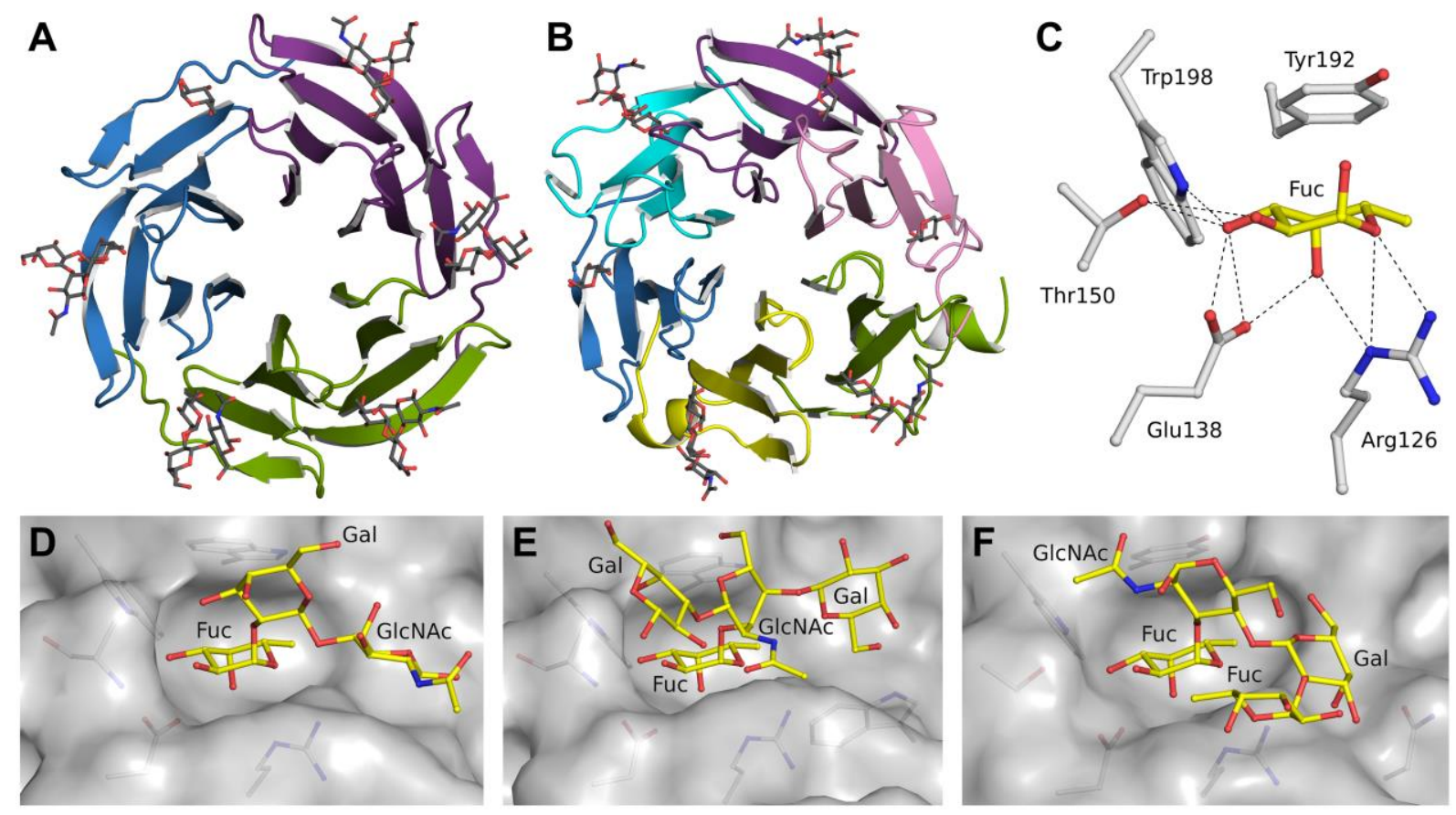

Figure 4 HBGA-pathogen interactions of selected airway infections. (A,B) Overall representations of the 6 -bladed $\beta$-propeller folds of BambL in complex with the H-type 2 determinant (A, PDB 3ZZV [60]) and AFL (FleA) with a blood group A determinant (B, PDB $4 \mathrm{AH} 4$ [63]). Coloring is by protein chain or blade, respectively. (C) Close-up view of the fucose binding site 3 in AFL. (D-F) Surface representations of the binding of H-type 2 (D; PDB 3ZZV [60]) and Lewis ${ }^{x}$ (E, PDB 3ZW1 [60]) determinants in the intramolecular binding site of BamBL, and Lewis ${ }^{y}$ (F, PDB 4D4U [63]) in binding site 3 of AFL. 